\title{
A theoretical model of the helium zone plate microscope
}

\author{
Adrià Salvador Palau, ${ }^{1}$ Gianangelo Bracco, ${ }^{1,2}$ and Bodil Holst ${ }^{1, *}$ \\ ${ }^{1}$ Department of Physics and Technology, University of Bergen, Allégaten 55, 5007 Bergen, Norway \\ ${ }^{2}$ CNR-IMEM, Department of Physics, University of Genova, V Dodecaneso 33, 16146 Genova, Italy
}

(Dated: January 8, 2017)

\begin{abstract}
Neutral helium microscopy is a new technique currently under development. Its advantages are the low energy, charge neutrality and inertness of the helium atoms, a potential large depth of field and the fact that at thermal energies the helium atoms do not penetrate into any solid material. This opens, among others, for the creation of an instrument that can measure surface topology on the nano scale even on surfaces with high aspect ratios. One of the most promising designs for helium microscopy is the zone plate microscope. It consists of a supersonic expansion helium beam collimated by an aperture (skimmer) focused by a Fresnel zone plate onto a sample. The resolution is determined by the focal spot size, which depends on the size of the skimmer, the optics of the system and on the velocity spread of the beam through the chromatic aberrations of the zone plate. An important factor for the optics of the zone plate is the width of the outermost zone, corresponding to the smallest opening in the zone plate. The width of the outermost zone is fabrication limited to around $10 \mathrm{~nm}$ with present day state of the art technology. Due to the high ionization potential of neutral helium atoms, it is difficult to build efficient helium detectors. Therefore it is crucial to optimize the microscope design to maximize the intensity for a given resolution and width of the outermost zone. So far this has not been addressed in the literature. Here we present an optimization model for the helium zone plate microscope. Assuming constant resolution and width of the outermost zone, we are able to reduce the problem to a two variable problem (zone plate radius and object distance) and we show that for a given beam temperature and pressure there is always a single intensity maximum. We compare our model with the highest resolution zone plate focussing images published and show that the intensity can be increased 7 times. Reducing the width of the outermost zone to $10 \mathrm{~nm}$ leads to an increase in intensity of more than 8000 times. Finally we show that with present day state of the art detector technology (ionisation efficiency $1 \times 10^{-3}$ ), a resolution of the order of $10 \mathrm{~nm}$ is possible. In order to make this quantification we have assumed a Lambertian reflecting surface and calculated the beam spot size that gives a signal $100 \mathrm{cts} / \mathrm{s}$ within a solid angle of $0.02 \mathrm{sr}$, following an existing helium microscope design.
\end{abstract}

\section{INTRODUCTION}

In a neutral helium microscope, short Nemi or SHeM (scanning helium microscope) a beam of neutral helium atoms is created by a supersonic expansion. An image is obtained by measuring either a reflected or transmitted signal as the helium beam is scanned across the sample. The energy of the beam is very low (less than $0.1 \mathrm{eV}$ for a wavelength of $0.1 \mathrm{~nm}$ ), which means that the atoms map the outermost electron density distribution of the sample and do not penetrate into solid material [1]. These properties make neutral helium microscopy suited for the investigation of nano-coatings, fragile and/or insulating surfaces and surface structures with high aspect ratios. By using for example a two detector setup it should even be possible to create a nano stereo microscope which can measure surface topography on the nano scale. If a small working distance is applied, the helium microscope can be used to investigate samples presently examined with scanning probe techniques, with the advantage that the helium beam offers completely standardised imaging properties.

The first neutral helium microscope images were published in 2008 [2]. They were transmission, shadow im-

* Corresponding author, Bodil.Holst@uib.no ages of porous structures, obtained by scanning a beam focussed down to $2 \mu \mathrm{m}$ using a Fresnel zone plate. Since then, two other research groups have managed to obtain helium microscope images in reflection with micron range resolution using pinhole microscopes [3-6]. The first reflection images were published in 2011 by Withman and Sanchez using a setup with just a pinhole and no skimmer [3]. This setup still claims the best resolution achieved so far with a helium microscope: $350 \mathrm{~nm}$ [7]. The theoretical resolution limit of a pinhole microscope is discussed in detail in [8].

The first helium atom focussing with a Fresnel zone plate was carried out by Carnal et al. using a beam of metastable helium [9]. Because the helium atoms do not penetrate into any solids, any transmission optical element used must be of the binary type with either completely transparent or completely opaque areas. The first focussing of a neutral, ground state helium beam with a zone plate was carried out by Doak et al. [10]. Both of these experiments used the classical Fresnel-Soret zone plate. The Beynon-Gabor zone plate, which is also binary, has been suggested as an alternative attractive candidate for focusing of helium atoms [11]. The main advantage of the Beynon-Gabor zone plate is that it has no higher order foci. Unfortunately it is very challenging to fabricate. In 2015 a new optical diffraction element "the atom sieve" was introduced and focussing down to 
$3.6 \mu \mathrm{m}$ diameter was demonstrated. The atom sieve was inspired by the photon sieve, which was invented in 2001 and originally intended for applications with soft X-rays [12]. It is a structure consisting of pinholes of varying size, arranged across the Fresnel zones in such a manner that it is possible to focus to a spot with a diameter smaller than the smallest pinhole. In addition, higher order diffraction and secondary maxima can be suppressed by several orders of magnitude. The original photon sieve as well as the first atom sieve are based on Fresnel-Soret zone plates. Other configurations have been suggested, for example, a Beynon-Gabor zone plate based photon sieve [13]. The great advantage of the atom sieve is that it is much easier to fabricate free standing holes than free standing rods, such as they are needed in a standard binary Fresnel zone plate. This is particularly important because the width of the outermost zone is a critical factor for the achievable resolution as will be discussed in the next section. It is reasonable to assume that fabrication of holes down to $10 \mathrm{~nm}$ should be possible [14].

A drawback of all zone plates is that only a fraction of the incident beam intensity goes into the first order focus. For the Fresnel-Soret zone plate as well as for the BeynonGabor zone plate the fraction is around 10\% (zone plate efficiency). This is discussed in detail in [11]. The transmission window for the atom sieve can be adjusted to yield an intensity which is at least half the intensity of a standard Fresnel-Soret zone plate [12]. Alternative zone plate designs, where the first zones are blocked to facilitate zone plate stability and filtering of the zero order diffraction term, typically have a transmitted intensity higher than the atom sieve but lower than the FresnelSoret zone plate [15]. A further drawback is chromatic aberration. This is particularly an issue for helium microscopy because the beam has a pressure dependent velocity spread. This is discussed in detail in this paper.

It should be mentioned that there is an alternative class of focussing element for neutral helium optics, namely mirrors [16-18]. The advantage of mirrors is that they have no chromatic aberrations so that the velocity spread of the beam does not play a role, though there will be a certain signal loss through diffraction and scattering from steps and point defects as well as inelastic scattering. The fabrication limit induced by the width of the outermost zone is also not an issue, so that the mirrors can in principle be made much larger. The use of graphene as a mirror coating gives an inert and very stable surface [19]. Quantum reflection for focusing has also been demonstrated [20]. Unfortunately, until now, it has not been possible to control the curvature of mirrors with high enough precision, so that Fresnel zone plate based optical elements remain the most promising approach for the focusing of neutral atom and molecular beams.

A diagram of a helium zone plate microscope can be found in Fig. 1. The basic idea is simple: the supersonic expansion helium beam is collimated by a conically shaped aperture, which we refer to as skimmer. An image of the skimmer opening is focussed onto a sample plane by a Fresnel zone plate. The resolution of the microscope is then determined by the focal spot size of the beam on the sample plane. In this work we define the resolution as the full width at half maximum intensity of the beam spot. The beam scattered off the sample is then collected at a given solid angle using a detector, and the variation in this signal, while the sample is scanned, is used to create an image. Alternatively the transmitted beam is detected by a detector placed behind a porous sample.

The aim of this paper is to determine the geometry of the zone plate system which gives the maximum signal intensity in the beam spot on the sample for a given resolution and given width of the outermost zone of the zone plate. Our basic assumption is that the beam is created in a supersonic expansion. For completeness we cite here the detailed description of how we model the source. The description is taken from [8]. In a supersonic expansion, the helium gas expands into vacuum through a nozzle that must have a diameter much bigger than the mean free path of the gas particles. The atoms then collide with each other until eventually collisions cease and the atoms are travelling in free molecular flow without interacting. The supersonic expansion is chosen over for example an effusive source because it gives the highest centre line intensity [21].

A common way to describe a supersonic expansion theoretically is the quitting surface model. Here, the spherical quitting surface represents the distance from the nozzle where the atoms have reached molecular flow and are no longer interacting [22]. The velocity distribution of the atoms along the surface can be described by the most probable velocity $\bar{v}$ along the parallel direction (the radial direction from the centre of propagation). This velocity is given together with either a single parallel temperature or, in a more detailed description, by a pair of temperatures $T_{\|}, T_{\perp}$ associated to the orthogonal components of the velocity in spherical coordinates. At the quitting surface, the perpendicular temperature, $T_{\perp}$ must be much smaller than the parallel temperature $T_{\|}$. In this paper, the conditions at the quitting surface are calculated solving Boltzmann equation [23-25]. Negligible collisional coupling is assumed at a distance where the temperatures of the beam fulfill $T_{\perp} / T_{\|} \leq 0.01$. Stopping the integration at $T_{\perp} / T_{\|}=0.005$ has shown to affect the flow parameters by less than $0.1 \%$ [26]. For a single temperature and constant density along the quitting surface, an analytical approximation for the intensity exists, obtained by Sikora in 1973 [22]. For a pair of temperatures, a numerical integral must be implemented [27]. From now on, we will name the single temperature solution Sikora's approximation and, following the convention in the literature, the dual temperature model ellipsoidal quitting surface model. Both models are explained in detail in Section II.

In praxis, one often measures an intensity reduction due to backscattering of atoms into the beam line. For a complete analysis, an optimization of the expansion pa- 
rameters (nozzle-skimmer distance, beam pressure and temperature etc.) is therefore important. This has been studied for example in [28-30] and is not a topic of this paper. We take all expansion parameters: the most probable velocity, the perpendicular and parallel temperatures of the beam, the skimmer-nozzle distance and the position of the quitting surface relative to the skimmer to be constant.

We set two further parameters to be constant: the resolution and the width of the outermost zone. The behaviour of the system with respect to the width of the outermost zone is monotone and easy to calculate if need be. This leaves us with four variables as can be seen from Fig. 1: The skimmer opening, $r_{\mathrm{S}}$, the zone plate radius $r_{\mathrm{zp}}$ the object distance, $a$, and the image distance, $b$. We show that this reduces to a two variable optimization problem using the optical equations of the system, which we describe in the next section. The intensity can then be calculated over a wide span of combinations and plotted in a single graph.

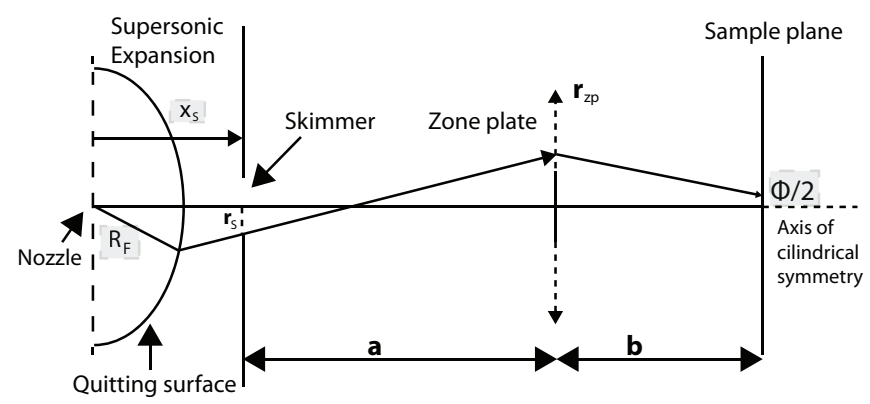

FIG. 1. Diagram of a zone plate microscope setup. The parameters kept constant are in grey boxes. $x_{\mathrm{S}}$ is the distance between the nozzle and the skimmer aperture, and $R_{\mathrm{F}}$ is the radius of the quitting surface. $b$ is the working distance, $\Phi$ is the focal spot size. $r_{\mathrm{S}}$ and $r_{\mathrm{zp}}$ are the radius of the skimmer and the radius of the zone plate respectively and $a$ is the distance between the skimmer and the zone plate. Note that the system is rotationally symmetrical about the main axis.

\section{THEORETICAL FOUNDATION}

\section{A. The optical system}

The size of the focal spot generated by a Fresnel zone plate by imaging an aperture of radius $r_{\mathrm{S}}$ is given by a convolution of the chromatic aberration term of the zone plate, $\sigma_{\mathrm{cm}}$, the aperture (here, the skimmer), and the Airy diffraction term $\sigma_{\mathrm{A}}[15]$. Both $\sigma_{\mathrm{cm}}$ and $\sigma_{\mathrm{A}}$ can be assumed to be Gaussian contributions, because the velocity spread is essentially Gaussian (eq. (14)) and the first ring of the Airy term approximates well to a Gaussian [31]. The skimmer is assumed to approximate to a step function and thus carries a $1 / \sqrt{3}$ term in the convolution $[8,15]$.

The assumption that the skimmer approximates to a step function gives a maximum bound to the focal spot size. In reality, the intensity distribution decreases significantly along the skimmer radius for large skimmers placed close to the quitting surface. This has been experimentally investigated in $[24,26]$. In this paper, we assume that the quitting surface is close enough to the skimmer so that the skimmer directly determines the object of the optical system. In general, for resolutions on the order of less than $1 \mu \mathrm{m}$, the skimmers needed for optimal design are sufficiently small compared to the radius of the quitting surface to justify such an assumption, regardless of how close the quitting surface is to the skimmer. It must be noted that the step function assumption is only used when determining the optic equations of the system. For the intensity model used in Sec. III to calculate the intensity of the focal spot, the real intensity profile and angular spread of the beam are considered with no further assumptions.

The standard deviation resulting from the convolution is multiplied by $2 \sqrt{2 \ln (2)}$ to obtain its Full Width at Half Maximum (FWHM):

$$
\begin{aligned}
\Phi=2 \sqrt{2 \ln (2)} \sqrt{\sigma_{\mathrm{cm}}^{2}+\sigma_{\mathrm{A}}^{2}+\left(\frac{M r_{\mathrm{S}}}{\sqrt{3}}\right)^{2}} \\
\equiv K \sqrt{\sigma_{\mathrm{cm}}^{2}+\sigma_{\mathrm{A}}^{2}+\left(\frac{M r_{\mathrm{S}}}{\sqrt{3}}\right)^{2}} .
\end{aligned}
$$

Where $\sigma_{\mathrm{cm}}$ is the chromatic aberration, and $\sigma_{\mathrm{A}}$ is the Airy diffraction term limiting the resolution of the microscope. The magnification factor, $M$, of the optical system greatly influences the focal spot size. It is given by the following equation [15]:

$$
M=\frac{b}{a}=\frac{f}{a-f} .
$$

Where $f$ is the focal length of the Fresnel zone plate which depends on its radius and $\Delta r$ as follows [15]

$$
f=\frac{2 r_{\mathrm{zp}} \Delta r}{\lambda} .
$$

Where $\lambda$ is the average de Broglie wavelength of the beam given by $\lambda=h / m \bar{v}, \mathrm{~m}$ is the mass of a helium atom, $h$ is the Planck constant and $\bar{v}$ is the most probable velocity of helium atoms along the radial direction. $b$ corresponds to the distance of the focused image from the zone plate, and is also the working distance of the microscope (Fig. 1). From combining eq. (2) and (3) while assuming constant $\Delta r$ and $\lambda$, one obtains $b \rightarrow b\left(a, r_{\mathrm{zp}}\right)$, reducing the system from four to three variables. The chromatic aberration term stems from the dependence of the focal length on the wavelength. It can be proven to be $[15,32]$ :

$$
\sigma_{\mathrm{cm}}=\frac{r_{\mathrm{zp}}}{S \sqrt{2}} .
$$

Where $S=\bar{v} / \sqrt{2 k_{\mathrm{B}} T_{||} / m}$ is the parallel speed ratio, which is used as a measure of the velocity spread of the 
beam. The Airy term stems from the diffraction of helium atoms with the Fresnel zone plate. It can be expressed in terms of the width of the smallest zone $\Delta r$ [15]:

$$
\sigma_{\mathrm{A}}=\frac{0.42 a \Delta r}{a-f}
$$

Where $a$ is the distance between the aperture and the zone plate. If $a \gg f$ the equation reduces to:

$$
\sigma_{\mathrm{A}}=0.42 \Delta r
$$

For the strong demagnification $(M \ll 1)$ desired in a zone plate microscope, this is the case (see eq. 2), and therefore eq. (6) will be used throughout the paper. This diffraction term will have a constant influence on the focal spot size. Therefore, it is convenient to define a corrected focal spot size $\Phi^{\prime}$, obtained by squaring eq. (1), subtracting $\sigma_{\mathrm{A}}^{2}$ and redefining the left hand side of the equation:

$$
\Phi^{\prime}=\sqrt{\frac{\Phi^{2}}{K^{2}}-\sigma_{\mathrm{A}}^{2}} .
$$

Eq. (1) now simplifies to:

$$
\Phi^{\prime 2}=\sigma_{\mathrm{cm}}^{2}+\left(\frac{M r_{\mathrm{S}}}{\sqrt{3}}\right)^{2}
$$

The optical system has three variables, the distance between the skimmer and the zone plate $a$, the radius of the skimmer $r_{\mathrm{S}}$ and the radius of the zone plate, $r_{\mathrm{zp}}$. For a given focal spot size $\Phi$, the variables are interrelated through eq. (1). In this paper we will mostly use the expression $r_{\mathrm{S}} \rightarrow r_{\mathrm{S}}\left(a, r_{\mathrm{ZP}}\right)$ :

$$
r_{\mathrm{S}}^{2}=\frac{3 \lambda^{2}}{4 \Delta r^{2}}\left(a-\frac{2 r_{\mathrm{zp}} \Delta r}{\lambda}\right)^{2}\left[\frac{\Phi^{\prime 2}}{r_{\mathrm{zp}}^{2}}-\frac{1}{2 S^{2}}\right]
$$

It is also interesting to obtain $a \rightarrow a\left(r_{\mathrm{S}}, r_{\mathrm{zp}}\right)$.

$$
a=f\left(r_{\mathrm{S}} \sqrt{\frac{1}{3\left(\Phi^{\prime 2}-\left(\frac{r_{\mathrm{zp}}}{S \sqrt{2}}\right)^{2}\right)}}+1\right) .
$$

This equation sets the expected limitations for a zone plate microscope. If the chromatic aberration and the Airy diffraction term add up to more than the desired focal spot, the solution is non-real and therefore the setup is non physical. Therefore eq. (10) sets a limit to the maximum physical zone plate radius for a given focal spot size. By imposing a real square root one obtains:

$$
r_{\mathrm{zp}} \leq \sqrt{2} S \Phi^{\prime}
$$

A Fresnel zone plate consists of a finite series of alternate zones. In the optimization presented in this paper, the radius of the zone plate is varied continuously from very small values. In practice, the finite value of the number of zones limits the zone plate radius step through the following equation [15]:

$$
N=\frac{\lambda f}{4 \Delta r^{2}}=\frac{r_{\mathrm{zp}}}{2 \Delta r}, \quad r_{\mathrm{zp}} \gg \Delta r .
$$

It is therefore important to keep this limitation in mind when designing a zone plate setup, we will see that for most cases of interest the assumption $r_{\mathrm{zp}} \gg \Delta r$ holds and it suffices to round the zone plate radius to a multiple of $\Delta r$. A detailed discussion regarding zone plates in the low $\mathrm{N}$ limit can be found in [39].

Eq. (11) also gives the theoretical minimum resolution of a zone plate microscope. By imposing that $\Phi^{\prime}$ is real one obtains:

$$
\frac{\Phi^{2}}{K^{2}} \geq \sigma_{\mathrm{A}}^{2}, \quad \Phi \gtrsim K \sigma_{\mathrm{A}} \approx \Delta r .
$$

Which means that the minimum resolution of a zone plate microscope is given by the smallest achievable pattern on a zone plate. This is a well known result previously derived for light optics [15].

\section{B. The intensity models}

As discussed in the introduction, the most general model of the intensity field of a supersonic helium beam is the ellipsoidal quitting surface model. The velocity distribution over the quitting surface is assumed to be elliptical Maxwell-Boltzmann [22]:

$$
\begin{aligned}
f_{\text {ell }}(\vec{v})= & n\left(\frac{m}{2 \pi k_{\mathrm{B}} T_{\|}}\right)^{\frac{1}{2}}\left(\frac{m}{2 \pi k_{\mathrm{B}} T_{\perp}}\right) . \\
& \exp \left(-\frac{m}{2 k_{\mathrm{B}} T_{\|}}\left(v_{\|}-\bar{v}\right)^{2}-\frac{m}{2 k_{\mathrm{B}} T_{\perp}} v_{\perp}^{2}\right) .
\end{aligned}
$$

Where $m$ is the mass of a helium atom, $k_{\mathrm{B}}$ is Boltzmann's constant and $T_{\|}$and $T_{\perp}$ are the parallel and perpendicular temperatures. $\bar{v}$ is the most probable velocity of the beam along the radial direction and $v_{\|}$and $v_{\perp}$ are the parallel and perpendicular components of the velocity, corresponding to the radial and angular components in spherical coordinates.

Integrating eq. (14) over the quitting surface, across the skimmer and over the zone plate surface, one obtains the following intensity equation:

$$
\begin{array}{r}
I_{\mathrm{E}}=\frac{\tau \eta I_{0}}{2 \pi a^{2} R_{\mathrm{F}}^{2} \mathrm{~L}} \int_{0}^{r_{\mathrm{zp}}} \int_{0}^{r_{\mathrm{S}}} \int_{0}^{\pi} g(\delta) r \cdot \rho \cos ^{3} \beta \cdot \epsilon^{3} \\
e^{-S^{2}\left(1-\epsilon^{2} \cos ^{2} \theta\right)} D(b) d \rho d r d \alpha .
\end{array}
$$

Where $R_{\mathrm{F}}$ is the radius of the quitting surface. $\eta=$ $1 / \pi^{2}$ is the geometrical efficiency of the zone plate for the first diffraction order, corresponding to about $10 \%$ of 
the incoming signal [15]. $I_{0}$ is the total intensity from a nozzle of diameter $d_{\mathrm{N}}[21]$ :

$$
I_{0}=\kappa \frac{P_{0}}{k_{\mathrm{B}} T_{0}} \sqrt{\frac{2 k_{\mathrm{B}} T_{0}}{m}}\left(\frac{\pi}{4} d_{\mathrm{N}}^{2}\right) \sqrt{\frac{\gamma}{\gamma+1}}\left(\frac{2}{\gamma+1}\right)^{1 /(\gamma-1)} .
$$

Where $P_{0}$ and $T_{0}$ are the thermodynamic pressure and temperature of the helium gas before it undergoes the expansion. $\gamma=\mathrm{C}_{\mathrm{P}} / \mathrm{C}_{\mathrm{V}}=5 / 3$ for helium. $\kappa=2$ is a peak factor as defined in [21]. All other parameters are defined in detail in Appendix B.

Unfortunately, the ellipsoidal quitting surface model has no simple analytical solutions and is often slow to compute over a wide space of solutions. Using the quitting surface model with a single radial velocity distribution it can be shown that for $S \geq 5, r_{\mathrm{S}} \ll R_{\mathrm{F}}-x_{\mathrm{S}}$, the intensity arriving at a zone plate small enough to justify a constant radial intensity distribution reads [22]:

$$
\begin{aligned}
& I_{\mathrm{S}}= \\
& \frac{I_{0} \eta \pi r_{\mathrm{zp}}^{2}}{\left(R_{\mathrm{F}}+a\right)^{2}}\left\{1-\exp \left[-S^{2}\left(\frac{r_{\mathrm{S}}\left(R_{\mathrm{F}}+a\right)}{R_{\mathrm{F}}\left(R_{\mathrm{F}}-x_{\mathrm{S}}+a\right)}\right)^{2}\right]\right\} .
\end{aligned}
$$

Where $R_{\mathrm{F}}-x_{\mathrm{S}}$ is the axial distance between the quitting surface and the tip of the skimmer. This equation was obtained independently by Sikora and Andersen [22] and thus we refer to it as Sikora's approximation as mentioned in the introduction. The radius of the skimmer, can be obtained from eq. (9), reducing the intensity equation to two independent variables:

$$
\begin{gathered}
I_{\mathrm{S}}\left(r_{\mathrm{zp}}, a\right)=\frac{I_{0} \eta \pi r_{\mathrm{zp}}^{2}}{\left(R_{\mathrm{F}}+a\right)^{2}}- \\
\frac{I_{0} \eta \pi r_{\mathrm{zp}}^{2}}{\left(R_{\mathrm{F}}+a\right)^{2}} \exp \left\{-\frac{3 \lambda^{2}}{4 \Delta r^{2}}\left(a-\frac{2 r_{\mathrm{zp}} \Delta r}{\lambda}\right)^{2}\left[\frac{\Phi^{\prime 2} S^{2}}{r_{\mathrm{zp}}^{2}}-\frac{1}{2}\right]\right. \\
\left.\left(\frac{\left(R_{\mathrm{F}}+a\right)}{R_{\mathrm{F}}\left(R_{\mathrm{F}}-x_{\mathrm{S}}+a\right)}\right)^{2}\right\} \cdot
\end{gathered}
$$

Assuming that $a$ is much bigger than the focal length, $f=\frac{2 r_{\mathrm{zp}} \Delta r}{\lambda}$, it follows that the maximum intensity of a helium beam monotonically increases with $1 / \Delta r$. Therefore, to obtain the maximum intensity, $\Delta r$ is chosen to be constant and equal to the smallest realisable value.

We can use this equation together with eq. (9) to obtain an analytical equation for the position of the intensity maxima given that either $a$ or $r_{\mathrm{zp}}$ is taken to be constant.

$$
\nabla I_{\mathrm{S}}\left(r_{\mathrm{zp}}, a\right)=(0,0), \quad \partial_{a}^{2} I<0, \quad \partial_{r_{\mathrm{zp}}}^{2} I<0 .
$$

Which corresponds to a subset of the solutions of the following equation:

$$
\nabla \frac{r_{\mathrm{zp}}^{2}}{\left(R_{\mathrm{F}}+a\right)^{2}}\left\{1-\exp \left[-S^{2}\left(\frac{r_{\mathrm{S}}\left(R_{\mathrm{F}}+a\right)}{R_{\mathrm{F}}\left(R_{\mathrm{F}}-x_{\mathrm{S}}+a\right)}\right)^{2}\right]\right\}
$$$$
=0 \text {. }
$$

The derivatives with respect to the zone plate radius and the skimmer-zone plate distance $a$ can both be solved analytically. The derivative of eq. (18) with respect to the skimmer-zone plate distance, $a$, is a simple cubic equation giving the $a$ corresponding to the maximum intensity at a given zone plate radius.

$$
\begin{array}{r}
a^{3}+2 a^{2}\left(R_{\mathrm{F}}-\sqrt{3 \Gamma} r_{\mathrm{zp}}\right)+a R_{\mathrm{F}}\left(R_{\mathrm{F}}-4 r_{\mathrm{zp}} \sqrt{3 \Gamma}\right) \\
=r_{\mathrm{zp}} \sqrt{3 \Gamma} R_{\mathrm{F}}^{2}\left[\frac{2 S^{2} \Phi^{\prime 2}+r_{\mathrm{zp}}^{2}(\Gamma-1)}{S^{2} \Phi^{\prime 2}-0.5 r_{\mathrm{zp}}^{2}}\right] .
\end{array}
$$

Where $\Gamma \equiv \frac{1}{3}\left(\frac{2 \Delta r}{\lambda}\right)^{2}$ is a constant of the problem which gives the relative size of the smallest aperture of the zone plate compared with the average wavelength of the beam, usually $\Gamma \gg 1$.

This approximation has been obtained under the following assumptions, all of them justified in Appendix A.

$$
\begin{gathered}
\frac{\left(R_{\mathrm{F}}+a\right)^{2}\left(a-\sqrt{3 \Gamma} r_{\mathrm{zp}}\right)\left[\frac{S^{2} \Phi^{\prime 2}}{r_{\mathrm{zp}}^{2}}-\frac{1}{2}\right]\left(a+\frac{R_{\mathrm{F}}}{a} \sqrt{3 \Gamma} r_{\mathrm{zp}}\right)}{\Gamma R_{\mathrm{F}}^{2} a^{2}} \ll 1, \quad(22) \\
a^{2}+R_{\mathrm{F}} r_{\mathrm{zp}} \sqrt{3 \Gamma} \approx a^{2}, \\
\left(a-\sqrt{3 \Gamma} r_{\mathrm{zp}}\right)^{2} \approx a\left(a-2 \sqrt{3 \Gamma} r_{\mathrm{zp}}\right), \\
R_{\mathrm{F}}-x_{\mathrm{S}} \ll a .
\end{gathered}
$$

$a^{\max }$ is therefore obtained by solving the cubic equality eq. (21) for $a$, which gives a single real positive solution. The explicit analytical expression for $a^{\max }$ has been included in appendix A. In this work, the cubic equation was solved numerically using MATLAB.

MATLAB was used to perform the numerical calculations in this work. Particularly the function roots was used to solve eq. (21) and integral3 was used to calculate the integral in eq. (15). In section III B, the $\max$ function was used to obtain several global maxima of Sikora's approximation, embedded within a "for loop".

\section{RESULTS AND DISCUSSION}

A. An example: $\Phi=0.9 \mu \mathrm{m}, \Delta r=323 \mathbf{n m}$

To illustrate the power of our model we consider some of the best results published so far of focussing helium with a zone plate [33]. Here a $0.9 \mu \mathrm{m}$ focus was achieved with, $a$ set to $1.528 \mathrm{~m}$ and $\Delta r$ to approximately $323 \mathrm{~nm}$ (from the two zone plates that were used, we choose to study the one referred as "the MIT zone plate"). In Fig. 2 we compare this setup to the best configuration achievable according to our model keeping the same focal 
spot size and $\Delta r$. The original setup is marked by a yellow cross, which an be seen to be placed far away from the intensity maximum (black cross). In Fig. 3 we show the best setup and best achievable intensity with the smallest realistic value of $\Delta r(10 \mathrm{~nm})$. In this case, the old setup cannot be compared in the same plot as $\Delta r$ has changed.

The intensity for the model calculations is computed using the ellipsoidal quitting surface model (eq. (15)) and plotted with respect to two variables: $r_{\mathrm{zp}}$ and $a$. The skimmer radius $r_{\mathrm{S}}$ is a hidden variable related to $r_{\mathrm{zp}}$ and $a$ by eq. (9). A clear intensity peak can be observed which for small zone plate radii follows well. The approximation given by eq. (21) (see Fig. 2 and 3 ), the approximation (solid line) is shown together with the real line of zero gradient of the ellipsoidal quitting surface model (dashed line).

If $\Delta r$ is set at a minimal construction limit of $10 \mathrm{~nm}$, the intensity increases significantly: 8000 times with respect to the original setup (see Fig. 3). In this case, eq. (21) gives a value very close to the position of the intensity peak calculated numerically.

The literature has so far featured relatively large zone plates $\left(r_{\mathrm{zp}}>90 \mu \mathrm{m}\right)$, a regime where Sikora's approximation can perform badly [8]. Therefore it is important to compare Sikora's approximation with the ellipsoidal quitting surface model. To do so, it is useful to plot the fraction of the normalised intensities:

$$
\zeta=\frac{I_{\mathrm{S}} \cdot \max \left(I_{\mathrm{E}}\right)}{I_{\mathrm{E}} \cdot \max \left(I_{\mathrm{S}}\right)}
$$

It can be seen that for zone plate microscopes, Sikora's approximation can be implemented across a broad zone plate range (Fig. 4). Interestingly, $\zeta$ is significantly bigger than 1 only for very small zone plates. This is the opposite behaviour than one would expect, but it can be understood when the skimmer radius is plotted. From the values of the skimmer radius one sees that the Sikora approximation fails for large radii, showing that the size of the aperture closer to the quitting surface (skimmer) is more important than the aperture further away (zone plate) (Fig. 5). This is due to the off-axis intensity decreasing faster the closer the quitting surface is to the plane of interest.

\section{B. Parametric dependences}

In this paper, we reduce a multi-variable multiparametric system to a two variable equation, giving a single optimal configuration for a zone plate microscope. In this section, we discuss the dependence of the system on some of the parameters considered constant. Particularly, we plot the dependence of the optimized system geometry $\left(\left(a^{\max }, r_{\mathrm{zp}}^{\max }, r_{\mathrm{S}}^{\max }\right)\right)$ and intensity I, as function of the focal spot size $\Phi$, the speed ratio $S$ and the width of the outermost zone $\Delta r$.
To do so, we use Sikora's approximation (eq. (18)), which approximates well the intensity maxima and requires much lower computation times than the ellipsoidal quitting surface model. The quitting surface properties such as the speed ratio (when constant) and the radius of the quitting surface are set at a fix value of $S=241.68$ and $R_{\mathrm{F}}=0.01129 \mathrm{~m}$, calculated solving the Boltzmann equation for $T_{0}=115 \mathrm{~K}, P_{0}=101 \mathrm{Bar}$ as described in the introduction.

From Fig. 6 we see that, as expected, a higher speed ratio yields a smaller optimal skimmer radius. This is because with higher speed ratios the beam is more collimated and thus increasing the skimmer radius affects the intensity less. We also see an increase of the intensity of the beam and an increase of both the zone plate radius and the distance $a$ (also a result of a more collimated beam). The increase of the optimal zone plate radius is correlated with the decrease in chromatic aberrations: bigger zone plates capture more particles but also have larger aberrations. Here, the increase in the speed ratio compensates the larger aberration term (see eq. (4)).

The dependence on the focal spot size, $\Phi$, is monotonous and increasing in all cases. As expected, when the focal spot increases, the maximum intensity increases as well (see Fig. 7).

As explained in Sec. III A, the maximum intensity increases dramatically with smaller $\Delta r$. This is due to a combination of a smaller microscope length with a larger zone plate radius.

To summarize: the theoretical best helium microscope design is a compact microscope with a relatively large zone plate, combining the closeness to the atom source with a large angle of collection (see Fig. 8).

\section{Realistic resolution limits}

In this section we estimate realistic resolution limits. The calculations are done by setting constraints to all variables, as discussed below. In a real microscope the angular distribution of the reflected intensity will depend on the sample topography. As a suitable reference we here assume Lambertian reflection. Further, we assume that the detector is placed at an angle of $\pi / 4$ radians relative to the sample normal with the detector opening area perpendicular to the detector angle. This is similar to what has been done in [8] where we model the resolution limits for a pinhole microscope.

The constraints we choose are a minimal working distance $b$ of $10 \mu \mathrm{m}$ (a value that has been claimed in literature [7]), and a minimal skimmer radius of $100 \mathrm{~nm}$, which is commercially available [34]. $\Delta r$ is set at $10 \mathrm{~nm}$ and the radius of the zone plate is limited to a minimum of $10 \Delta r . a$ is set to a minimum of $1 \mathrm{~mm}$.

A minimal count rate of 100 counts per second is chosen. This is to a certain extend an arbitrary choice, but $100 \mathrm{cts} / \mathrm{s}$ for a Lambertian surface, ensures a reasonably large range of measurable intensity variations from differ- 


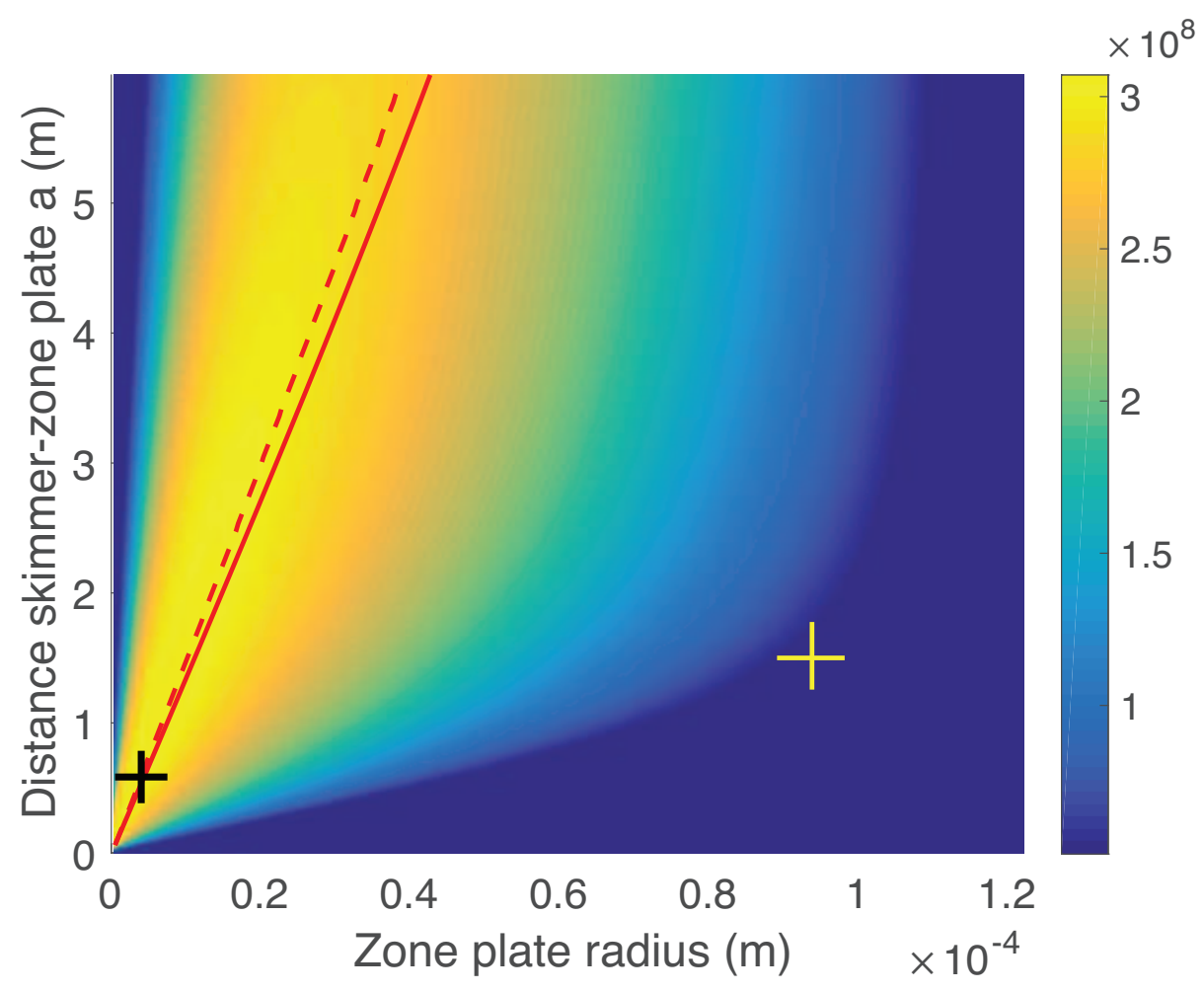

FIG. 2. Intensity (part/s) in a $0.9 \mu \mathrm{m}$ focal spot of a zone plate helium microscope for a span of values of $r_{\mathrm{zp}}$ and $a$ and for a constant $\Delta r=323 \mathrm{~nm}$. The solid line shows the subset of maximum solutions given by eq. (21), and the dashed line shows the numerical solution using the ellipsoidal quitting surface model. The intensity was calculated using the ellipsoidal quitting surface model with the following parameters: $T_{\|}=0.0052 \mathrm{~K}, T_{\perp}=0.0035 \mathrm{~K}, R_{\mathrm{F}}=0.01129 \mathrm{~m}, x_{\mathrm{S}}=0.0113 \mathrm{~m}$, $T_{0}=115 \mathrm{~K}, P_{0}=101$ Bar, $\lambda=0.089 \mathrm{~nm}$. The nozzle diameter $d_{\mathrm{n}}$ is set at $10 \mu \mathrm{m}$. The maximum (black cross) lies at $\left(a^{\max }, r_{\mathrm{zp}}^{\max }, r_{\mathrm{S}}^{\max }\right)=\left(0.555 \mathrm{~m}, 3.763 \times 10^{-6} \mathrm{~m}, 1.195 \times 10^{-5} \mathrm{~m}\right)$. The yellow cross indicates the configuration corresponding to the original setup used in [33]. The optimized design would increase the intensity by 7 times. An intensity increase of as much as 8000 times can be achieved by reducing $\Delta r$ to $10 \mathrm{~nm}$ (see Fig. 3).

ent surface topographies provided the background signal can be kept low. The count rate is determined by calculating the beam intensity using Sikora's approximation followed by deflection by a Lambertian surface, as follows:

$$
N=\frac{I_{\mathrm{s}}^{\text {optim }} R^{2}}{d^{2}} \eta_{\mathrm{D}} \cos \left(\frac{\pi}{4}\right)^{2} \approx 1.4 \times 10^{-7} I_{\mathrm{s}}^{\text {optim }} .
$$

Where $d=3 \mathrm{~mm}$ is the distance between the sample surface and the detector, $R=0.5 \mathrm{~mm}$ is the radius of the opening area in the detector. This corresponds to the solid angle of $0.02 \pi$ sr mentioned in the abstract, which was chosen because it corresponds to the solid angle used in the most recently published pinhole microscope experiment by Barr et al. [30]. We have also used similar beam parameters. $I_{\mathrm{s}}^{\text {optim }}$ is the optimized intensity using Sikora's approximation. $\eta_{\mathrm{D}}$ is the efficiency of the detector, chosen to be between $10^{-3}$ and $10^{-5}$ (an upper limit estimate for a commercial mass spectrometer) [35-38].

As we can see by comparing Fig. 7 and Fig. 9, resolutions close to the diffraction limit $(10 \mathrm{~nm})$ are possible for a very small working distance and the most efficient detector. Such a configuration usually requires a small number of zones. Even for a small number of zones (more than five) the resolution has been shown to approximate well to the optic lens limit and thus the result should be correct to within few nanometers [39]. For a detector of $\eta_{\mathrm{D}}=10^{-5}$, a microscope with a resolution of $30 \mathrm{~nm}$ is possible. In this case, the number of zones used in practical setups is already large enough to be in the lens approximation regime.

\section{A realistic "best resolution" configuration}

The construction limits considered in the previous section are arguably not stringent enough. Although a separation of $1 \mathrm{~mm}$ between the skimmer and the zone plate is possible, placing a vacuum pump within that distance is difficult. Similarly, a working distance of only $10 \mu \mathrm{m}$, although demonstrated, is technically challenging to implement in the reflection mode, and limits the samples that can be imaged. Therefore, we have also calculated the resolution limit with a minimum $a=5 \mathrm{~cm}$ and a minimum working distance, $b$, of $3 \mathrm{~mm}$ to set a realistic limit. 


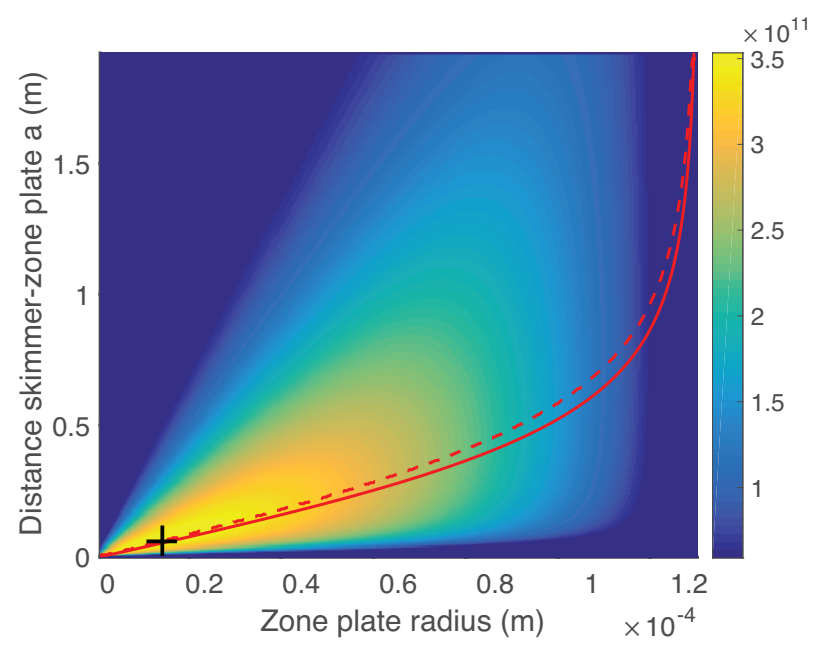

FIG. 3. Intensity (part/s) in a $0.9 \mu \mathrm{m}$ focal spot of a zone plate helium microscope for a span of values of $r_{\mathrm{zp}}$ and $a$ and for a constant $\Delta r=10 \mathrm{~nm}$. The red line shows the subset of maximum solutions given by eq. (21) and the dashed line shows the real line of zero derivative in the ellipsoidal quitting surface model. The intensity was calculated using the ellipsoidal quitting surface model with the following parameters: $T_{\|}=0.0052 \mathrm{~K}, T_{\perp}=0.0035 \mathrm{~K}, R_{\mathrm{F}}=0.01129 \mathrm{~m}, x_{\mathrm{S}}=0.0113$ $\mathrm{m}, T_{0}=115 \mathrm{~K}, P_{0}=101 \mathrm{Bar}, \lambda=0.089 \mathrm{~nm}$. The nozzle diameter $d_{\mathrm{n}}$ is set at $10 \mu \mathrm{m}$. The maximum (black cross) lies at $\left(a^{\max }, r_{\mathrm{zp}}^{\max }, r_{\mathrm{S}}^{\max }\right)=\left(0.06 \mathrm{~m}, 12.22 \times 10^{-6} \mathrm{~m}, 1.173 \times 10^{-5} \mathrm{~m}\right)$. Note how the intensity peak is at $3.5 \times 10^{11}$ (part/s), corresponding to an intensity of about 8000 times the configuration used in [33]

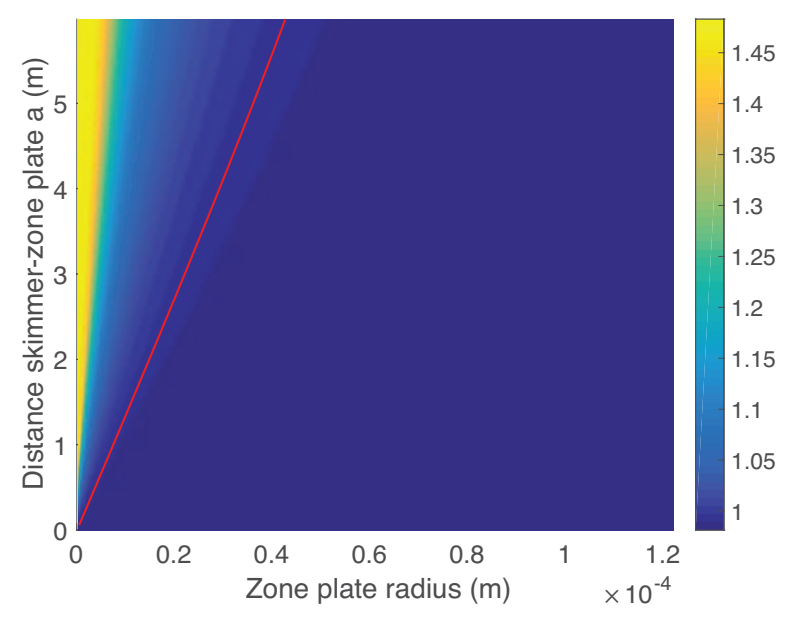

FIG. 4. Normalised fraction of Sikora's model divided by the ellipsoidal quitting surface model, $\zeta$, for a span of values of $r_{\mathrm{zp}}$ and $a$. The solution was calculated for a zone plate helium microscope with a resolution of $0.9 \mu \mathrm{m}$ and a $\Delta r$ of $323 \mathrm{~nm}$. The red line shows the subset of maximum solutions given by eq. (21). Both models were computed using the following parameters: $T_{\|}=0.0052 \mathrm{~K}, T_{\perp}=0.0035 \mathrm{~K}, R_{\mathrm{F}}=0.01129$ $\mathrm{m}, x_{\mathrm{S}}=0.0113 \mathrm{~m}, T_{0}=115 \mathrm{~K}, P_{0}=101 \mathrm{Bar}, \lambda=0.089 \mathrm{~nm}$.

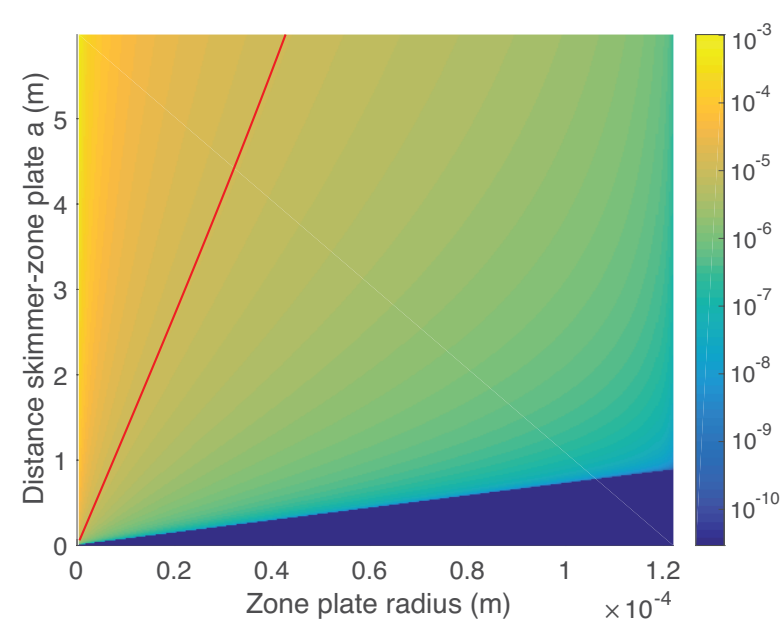

FIG. 5. Skimmer radius $r_{\mathrm{S}}[\mathrm{m}]$, for a zone plate helium microscope with a resolution of $0.9 \mu \mathrm{m}$ and a $\Delta r$ of $323 \mathrm{~nm}$. The radius is plotted with a logarithm scale due to the high variations in its magnitude. Note how in the areas where Sikora's approximation fails (see Fig. 4), the skimmer radius is largest. The red line indicates the subset of maximum solutions given by eq. (21). The radius was calculated using the following parameters: $S=241.68, \lambda=8.9 \times 10^{-11} \mathrm{~m}$.

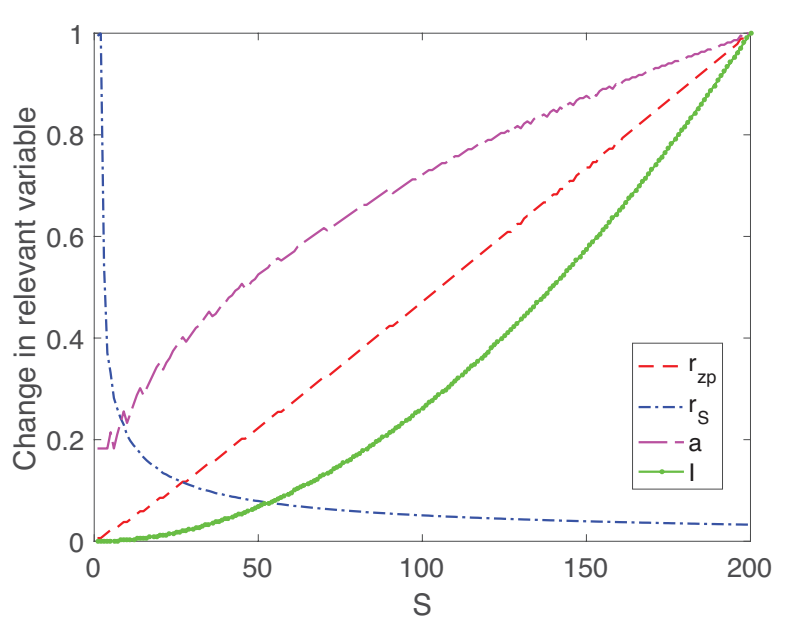

FIG. 6. Normalised optimized parameters of a zone plate helium microscope for a span of values of the speed ratio $S$. The intensity was calculated using Sikora's approximation with the following parameters: $T_{0}=115 \mathrm{~K}, P_{0}=101$ Bar at a fix resolution, $\Phi=0.9 \mu \mathrm{m}$ and $\Delta r=10 \mathrm{~nm}$. The maximum values of the parameters, used for normalization are: $r_{\mathrm{zp}}=10.2 \mu \mathrm{m}, r_{\mathrm{S}}=458.6 \mu \mathrm{m}, a=0.05 \mathrm{~m}, I=2.65 \times 10^{11}$ part/s. The small fluctuation of the data are due to numerical effects.

For $\eta_{\mathrm{D}}=10^{-5}$, such a microscope has a resolution limit of about $100 \mathrm{~nm}$. For $\lambda=0.088 \mathrm{~nm}$, the associated skimmer radius is $0.8 \mu \mathrm{m}$. The zone plate radius is $12.4 \mu \mathrm{m}$, $\Delta r$ is $10 \mathrm{~nm}$. For $\eta_{\mathrm{D}}=10^{-3}$, the resolution is limited to $80 \mathrm{~nm}$ by eq. (10) and not by the 100 counts/s limita- 


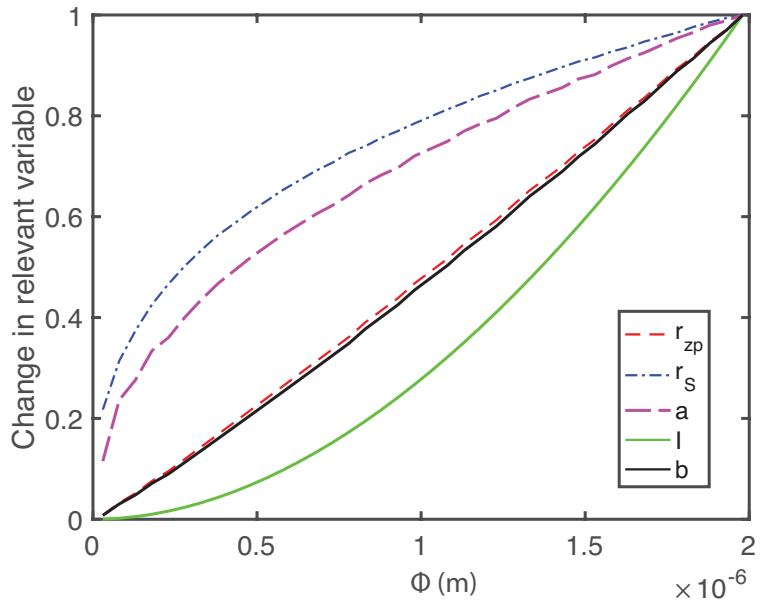

FIG. 7. Normalised optimized parameters of a zone plate helium microscope for a span of values of the focal spot size $\Phi$ for a fix $\Delta r=10 \mathrm{~nm}$. The intensity was calculated using Sikora's approximation with the following parameters: $T_{0}=$ $115 \mathrm{~K}, P_{0}=101 \mathrm{Bar}$, which corresponds to $S=241.7$. The maximum values of the parameters, used for normalization are: $r_{\mathrm{zp}}=29.7 \mu \mathrm{m}, r_{\mathrm{S}}=17.4 \mu \mathrm{m}, a=0.09 \mathrm{~m}, I=1.68 \times 10^{12}$ part $/ \mathrm{s}, b=0.007 \mathrm{~m}$. The small fluctuation of the data are due to numerical effects.

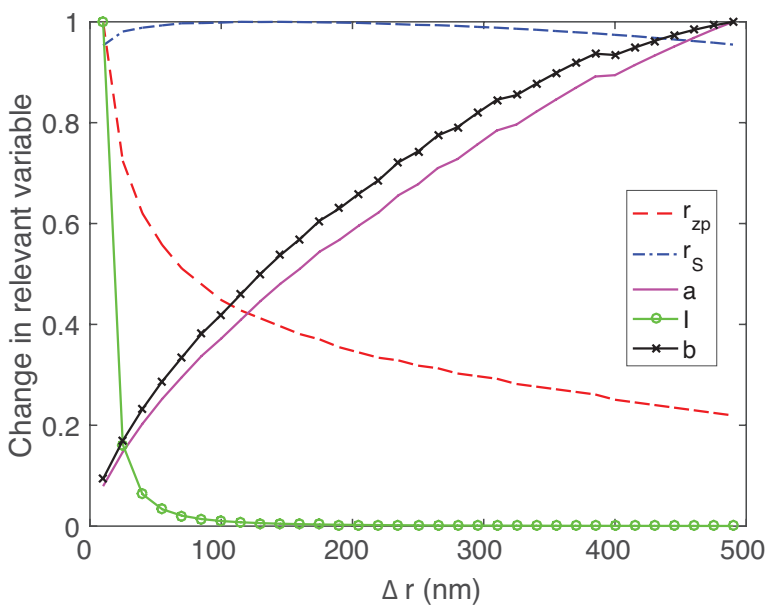

FIG. 8. Normalised optimized parameters of a zone plate helium microscope for a span of values of the width of the outermost zone $\Delta r$. The intensity was calculated using Sikora's approximation with the following parameters: $T_{0}=115 \mathrm{~K}$, $P_{0}=101$ Bar at a fix resolution $\Phi=0.9 \mu \mathrm{m}$. This corresponds to a speed ratio of $S=241.7$. In this case, $b$ has also been included to emphasize the reduction of the microscope length at high intensity setups. The maximum values of the parameters, used for normalization are: $r_{\mathrm{zp}}=12.5 \mu \mathrm{m}$, $r_{\mathrm{S}}=13.9 \mu \mathrm{m}, a=0.7515 \mathrm{~m}, I=3.81 \times 10^{11} \mathrm{part} / \mathrm{s}$, $b=0.0316 \mathrm{~m}$. The small fluctuation of the data are due to numerical effects.

tion. This later case corresponds to a $0.2 \mu \mathrm{m}$ skimmer radius and the same values for the rest of variables.

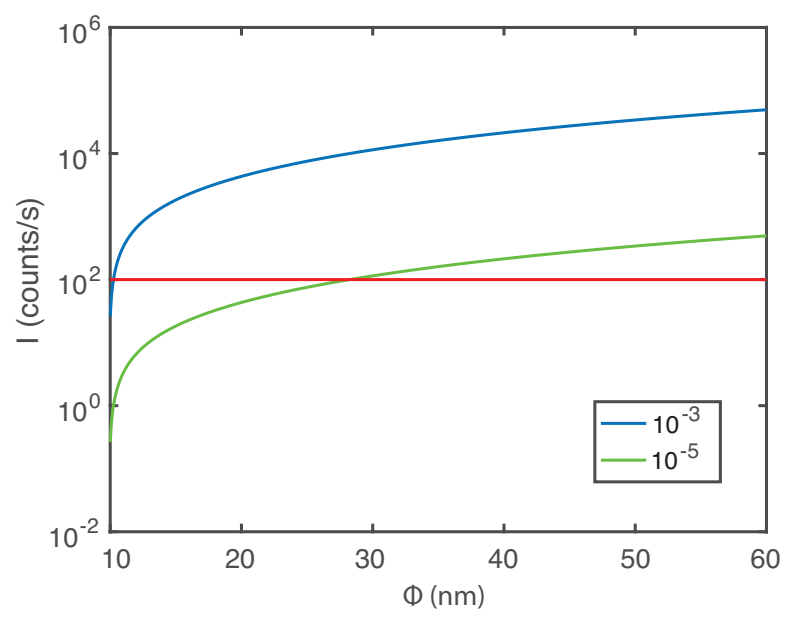

FIG. 9. Optimized count rate for different focal spot sizes. The intensity was calculated using Sikora's approximation with the following parameters: $T_{0}=115 \mathrm{~K}, P_{0}=161$ Bar, which corresponds to $S=241.7$. The efficiency of the detector, placed at $\pi / 4$ radians is $\eta=1 \times 10^{-5}$ (lower line) or $\eta=1 \times 10^{-3}$ (upper line). The red line indicates the 100 part/s intensity limit. The constraints on the calculation are a minimum working distance of $10 \mu \mathrm{m}$, a minimum skimmer radius of $100 \mathrm{~nm}$ and a minimum $a$ of $1 \mathrm{~mm} . \Delta r$ is set at 10 nm.

\section{CONCLUSION}

In this paper, we present a theoretical model of the neutral helium zone plate microscope. Using the optical equations of the system and the quitting surface approach, we are able to obtain a two variable intensity function for a given microscope resolution and width of the outermost zone of the zone plate. This function shows a clear intensity peak from which the best zone plate microscope design can be determined. By imposing realistic design constraints, we find that zone plate microscopes with a resolution as low as $10 \mathrm{~nm}$ are possible. We show how our model can be used to increase the intensity of a published setup by as much as 8000 times. The approach followed in this paper can easily be implemented for different intensity models, such as models describing other types of molecular beams and/or considering backscattering from the skimmer, by simply adapting the intensity equation.

\section{ACKNOWLEDGMENTS}

The work presented here was sponsored by the European Union: Theme NMP.2012.1.4-3 Grant no. 309672, project NEMI (Neutral Microscopy).

We thank Arnau Jiménez Castany and Kristoffer Johansen for their useful insights in the definition of eq. (19). 


\section{APPENDIX A: DERIVATION OF EQ. (21)}

To obtain eq. (21), one must derive eq. (18) with respect to the skimmer-zone plate distance $a$ and set it equal to 0 . One then obtains (assuming $R_{\mathrm{F}}-x_{\mathrm{S}} \ll a$ ):

$$
\begin{aligned}
& 1=\exp (-F) \cdot(1+ \\
& \left.\frac{\left(\frac{S^{2} \Phi^{\prime 2}}{r_{z \mathrm{p}}^{2}}-\frac{1}{2}\right)\left(R_{\mathrm{F}}+a\right)^{2}\left(a-\sqrt{3 \Gamma} r_{\mathrm{z}}\right)\left[a^{2}+R_{\mathrm{F}} r_{\mathrm{zp}} \sqrt{3 \Gamma}\right]}{\Gamma R_{\mathrm{F}}^{2} a^{3}}\right)
\end{aligned}
$$

With

$$
F \equiv \frac{\left(\frac{S^{2} \Phi^{\prime 2}}{r_{\mathrm{zp}}^{2}}-\frac{1}{2}\right)\left(R_{\mathrm{F}}+a\right)^{2}\left(a-\sqrt{3 \Gamma} r_{\mathrm{zp}}\right)^{2}}{\Gamma R_{\mathrm{F}}^{2} a^{2}} .
$$

Eq. (A1) can be written as follows:

$$
\exp (-F)=\frac{1}{1+A}=1+\varpi, \quad \varpi \equiv \frac{-A}{1+A} .
$$

with

$$
\begin{aligned}
& A= \\
& \frac{\left(\frac{S^{2} \Phi^{\prime 2}}{r_{z \mathrm{p}}^{2}}-\frac{1}{2}\right)\left(R_{\mathrm{F}}+a\right)^{2}\left(a-\sqrt{3 \Gamma} r_{\mathrm{zp}}\right)\left[a^{2}+R_{\mathrm{F}} r_{\mathrm{zp}} \sqrt{3 \Gamma}\right]}{\Gamma R_{\mathrm{F}}^{2} a^{3}} .
\end{aligned}
$$

By plotting A along a wide range of parameters one can see that for the conditions of interest along the intensity maxima $A \ll 1$. By taking the logarithm in eq. (A3) and expanding using Taylor series $\log (1-A) \approx-A$, one obtains:

$$
F \approx \frac{A}{1+A}
$$

Which reduces to:

$$
\left(a-\sqrt{3 \Gamma} r_{\mathrm{zp}}\right)(1+A)=\frac{a^{2}+R_{\mathrm{F}} r_{\mathrm{zp}} \sqrt{3 \Gamma}}{a} .
$$

In general, the radius of the quitting surface, $R_{\mathrm{F}}$, is of the order of millimetres (for the example shown in Sec. III C it corresponds to around $10 \mathrm{~mm}$ ). For microscopes with low resolution and realistic speed ratios of the order of 100 , the radius of the zone plate is as much hundreds of times the focal spot size (see eq. (11)). Conservatively, this means $\mathrm{O}\left(r_{\mathrm{zp}}\right) \approx 1 \times 10^{-4}$. The product of the zone plate radius and the radius of the quitting surface is then of the order of $R_{\mathrm{F}} r_{\mathrm{zp}}<1 \times 10^{-6} \cdot \sqrt{\Gamma}$ scales as $\Delta r / \lambda$, which for high $\Delta r$ is on the order of a few thousand. When compared with the order of $a^{2}, \mathrm{O}\left(a^{2}\right) \approx 1 \times 10^{-2}$, the product $R_{\mathrm{F}} r_{\mathrm{zp}} \sqrt{3 \Gamma}$ is at least one order of magnitude smaller (in practise in the studied cases, it was at least two orders of magnitude smaller). Therefore we can make the following approximation:

$$
a^{2}+R_{\mathrm{F}} r_{\mathrm{zp}} \sqrt{3 \Gamma} \approx a^{2} .
$$

When developing eq. (A5), we will encounter ( $a-$ $\left.\sqrt{3 \Gamma} r_{\mathrm{zp}}\right)$ at first and second exponent. It will be helpful to find some approximation to it. $\sqrt{3 \Gamma} r_{\mathrm{zp}}$ is smaller than $a$ such that the following approximation holds:

$$
\begin{aligned}
& \left(a-\sqrt{3 \Gamma} r_{\mathrm{zp}}\right)^{2}=a^{2}-2 \sqrt{3 \Gamma} r_{\mathrm{zp}} a+3 \Gamma r_{\mathrm{zp}}^{2} \approx \\
& a^{2}-2 \sqrt{3 \Gamma} r_{\mathrm{zp}} a=a\left(a-2 \sqrt{3 \Gamma} r_{\mathrm{zp}}\right) .
\end{aligned}
$$

Implementing these approximations in eq. (A5) one obtains:

$$
\frac{\left(\frac{S^{2} \Phi^{\prime 2}}{r_{\mathrm{zp}}^{2}}-\frac{1}{2}\right)\left(R_{\mathrm{F}}+a\right)^{2}\left(a-2 \sqrt{3 \Gamma} r_{\mathrm{zp}}\right)}{\Gamma R_{\mathrm{F}}^{2}}=\sqrt{3 \Gamma} r_{\mathrm{zp}} .
$$

By multiplying by $r_{\mathrm{zp}}^{3}$ and grouping for powers of $a$, one recovers eq. (21):

$$
\begin{aligned}
a^{3}+2 a^{2} & \left(R_{\mathrm{F}}-\sqrt{3 \Gamma} r_{\mathrm{zp}}\right)+a R_{\mathrm{F}}\left(R_{\mathrm{F}}-4 r_{\mathrm{zp}} \sqrt{3 \Gamma}\right) \\
& =r_{\mathrm{zp}} \sqrt{3 \Gamma} R_{\mathrm{F}}^{2}\left[\frac{2 S^{2} \Phi^{\prime 2}+r_{\mathrm{zp}}^{2}(\Gamma-1)}{S^{2} \Phi^{\prime 2}-0.5 r_{\mathrm{zp}}^{2}}\right] .
\end{aligned}
$$

The second derivative of eq. (18) with respect to $a$ is too lengthy to justify an analytical proof of the negative value of the second derivative at the intensity maximum. Instead, we follow a more practical numerical approach, which confirmed that the only real positive solution of eq. (A10) evaluates to a negative second derivative of eq. (18).

The analytical solution of eq. (A10) follows:

$$
\begin{aligned}
& a=\left(\frac{\sqrt{27 I^{2}+\left(18 G H-4 G^{3}\right) I+4 H^{3}-G^{2} H^{2}}}{23^{\frac{3}{2}}}-\right. \\
& \left.\frac{-27 I-9 G H+2 G^{3}}{54}\right)^{\frac{1}{3}} \\
& +\frac{G^{2}-3 H}{9\left(\frac{\sqrt{27 I^{2}+\left(18 G H-4 G^{3}\right) I+4 H^{3}-G^{2} H^{2}}}{23^{\frac{3}{2}}}-\frac{-27 I-9 G H+2 G^{3}}{54}\right)^{\frac{1}{3}}}-\frac{G}{3} .
\end{aligned}
$$

Where $G \equiv 2\left(R_{\mathrm{F}}-\sqrt{3 \Gamma} r_{\mathrm{zp}}\right), \quad H \equiv R_{\mathrm{F}}\left(R_{\mathrm{F}}-\right.$ $\left.4 r_{\mathrm{zp}} \sqrt{3 \Gamma}\right)$ and $I \equiv r_{\mathrm{zp}} \sqrt{3 \Gamma} R_{\mathrm{F}}^{2}\left[\frac{2 S^{2} \Phi^{\prime 2}+r_{\mathrm{zp}}^{2}(\Gamma-1)}{S^{2} \Phi^{\prime 2}-0.5 r_{\mathrm{zp}}^{2}}\right]$.

\section{APPENDIX B: THE ELLIPSOIDAL QUITTING SURFACE MODEL}

The ellipsoidal quitting surface model used in this paper is an extension of Sikora's ellipsoidal quitting surface 
model except that the skimmer is allowed to be in a different position than the quitting surface. Such a model was published by U. Bossel in 1974 [27]. Unfortunately the original paper has a couple of typos so we describe here the corrected equations in detail. The main intensity equation arriving at the zone plate is eq. (15):

$$
\begin{array}{r}
I_{\mathrm{E}}=\frac{\tau I_{0}}{2 \pi a^{2} R_{\mathrm{F}}^{2} \mathrm{~L}} \int_{0}^{r_{\mathrm{zp}}} \int_{0}^{r_{\mathrm{S}}} \int_{0}^{\pi} g(\delta) r \cdot \rho \cos ^{3} \beta \cdot \epsilon^{3} \\
e^{-S^{2}\left(1-\epsilon^{2} \cos ^{2} \theta\right)} D(b) d \rho d r d \alpha .
\end{array}
$$

Where $R_{\mathrm{F}}$ is the radius of the quitting surface and $S=\bar{v} / \sqrt{2 k_{\mathrm{B}} T_{\|} / m}$ is the parallel speed ratio. $\epsilon \equiv$ $\left(\left(\tau \sin ^{2} \theta+\cos ^{2} \theta\right)^{-1 / 2}, \tau \equiv \frac{T_{\|}}{T_{\perp}}\right.$ are auxiliary functions. The function $\mathrm{D}(\mathrm{b})$ is defined as follows:

$D(b) \equiv \frac{2}{\sqrt{\pi}} b e^{-b^{2}}+\left(2 b^{2}+1\right)[1+\operatorname{erf}(b)], \quad b \equiv S \epsilon \cos \theta$.

Where $I_{0}$ is defined in eq. (16). $g(\delta)$ is obtained from the angular term in eq. (12) of Beijerinck and Verster paper [21] (in Bossel's work a $\cos ^{2}$ function is chosen instead $[27])$ :

$$
g(\delta)=\cos ^{3}\left(\frac{\pi \theta}{2 \theta_{0}}\right), \quad \theta_{0}=\frac{\pi}{2} \sqrt{\frac{\gamma+1}{\gamma-1}-1}=\frac{\pi}{2} .
$$

Where $\gamma=C_{\mathrm{P}} / C_{\mathrm{V}}=5 / 3$ for helium has been used. L corresponds to the integration of $g(\delta)$ along the half sphere (all the intensity emitted by the source is set to be contained in $g(\delta))$.

$$
L \equiv \int_{0}^{\frac{\pi}{2}} g(\delta) \sin \delta d \delta=\frac{1}{4}
$$

$\rho$ is the radial coordinate at the zone plate plane and $r$ is the radial coordinate at the skimmer plane. The angles $\beta, \alpha$ and $\theta$ are shown in Fig. 10. The analytical expressions that relate them to the radial coordinates of the system follow:

$$
\begin{gathered}
\cos \beta=\frac{a}{\sqrt{a^{2}+(r \sin \alpha)^{2}+(\rho-r \cos \alpha)^{2}}}, \quad a=x_{\mathrm{D}}-x_{\mathrm{S}} \\
\cos \theta=\frac{x_{\mathrm{R}}\left(x_{\mathrm{D}}-x_{\mathrm{R}}\right)-y_{\mathrm{R}}^{2}+z_{\mathrm{R}}\left(\rho-z_{\mathrm{R}}\right)}{R_{\mathrm{F}} \sqrt{\left(x_{\mathrm{D}}-x_{\mathrm{R}}\right)^{2}+y_{\mathrm{R}}^{2}+\left(\rho-z_{\mathrm{R}}\right)^{2}}} .
\end{gathered}
$$

Where:

$$
\begin{gathered}
x_{\mathrm{R}}=x_{\mathrm{D}}-\xi\left(x_{\mathrm{D}}-x_{\mathrm{S}}\right), \quad y_{\mathrm{R}}=r \sin \alpha\left(\frac{x_{\mathrm{D}}-x_{\mathrm{R}}}{x_{\mathrm{D}}-x_{\mathrm{S}}}\right), \\
z_{\mathrm{R}}=r \cos \alpha\left(\frac{x_{\mathrm{D}}-x_{\mathrm{R}}}{x_{\mathrm{D}}-x_{\mathrm{S}}}\right)-\rho\left(\frac{x_{\mathrm{D}}-x_{\mathrm{R}}}{x_{\mathrm{D}}-x_{\mathrm{S}}}\right)+\rho,
\end{gathered}
$$

are the Cartesian coordinates of a point $\mathbf{P}$ on the quitting surface (see Fig. 10).

$$
\xi=\frac{B-\sqrt{B^{2}-A C}}{A} .
$$

$$
\begin{gathered}
A \equiv\left(x_{\mathrm{D}}-x_{\mathrm{S}}\right)^{2}+(r \sin \alpha)^{2}+(\rho-r \cos \alpha)^{2}, \\
B=x_{\mathrm{D}}\left(x_{\mathrm{D}}-x_{\mathrm{S}}\right)+\rho(\rho-r \cos \alpha) \\
C=x_{\mathrm{D}}^{2}+\rho^{2}-R_{\mathrm{F}}^{2} .
\end{gathered}
$$

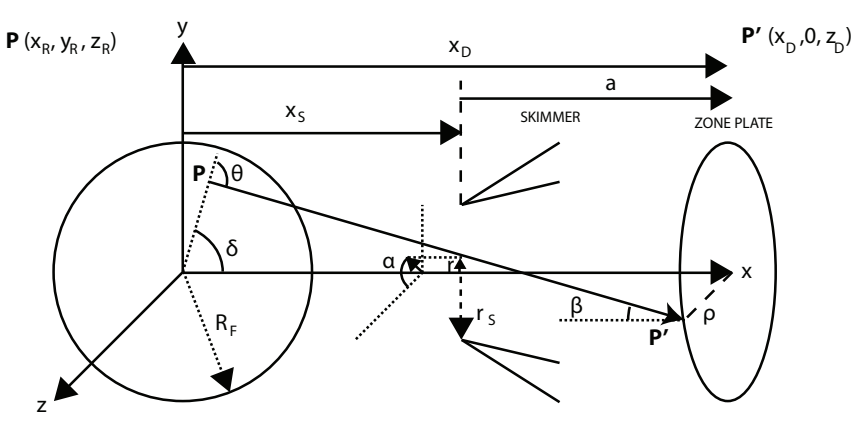

FIG. 10. Illustration of all variables used in the ellipsoidal quitting surface model. $\mathbf{P}$ is a point on the quitting surface from which a particle leaves in a straight trajectory until $\mathbf{P}$, a point placed on the zone plate plane. The point on the quitting surface is given by the set of Cartesian coordinates $\left(x_{\mathrm{R}}, y_{\mathrm{R}}, z_{\mathrm{R}}\right)$, which can be related to the polar coordinates $r, \alpha, \rho$ for further integration. $x_{\mathrm{S}}$ is the distance from the nozzle to the skimmer and $x_{\mathrm{D}}$ is the distance from the nozzle to the zone plate. Therefore $a=x_{\mathrm{D}}-x_{\mathrm{S}}$. The angles $\beta$ and $\theta$ can also be expressed in terms of $r, \alpha$ and $\rho$.
[1] G. Bracco and B. Holst, "Surface Science Techniques," (Springer, 2013).

[2] M. Koch, S. Rehbein, G. Schmahl, T. Reisinger, G. Bracco, W. E. Ernst, and B. Holst, J. Microsc. 229, 1 (2008).

[3] P. Witham and E. Sanchez, Rev. Sci. Instrum. 82, 103705 (2011).
[4] P. Witham and E. Sanchez, Cryst. Res. Technol. 29, 690 (2014).

[5] A. Fahy, M. Barr, J. Martens, and P. Dastoor, Rev. Sci. Instrum 86, 023704 (2015).

[6] M. Barr, A. Fahy, J. Martens, A. P. Jardine, D. J. Ward, J. Ellis, W. Allison, and P. C. Dastoor, Nat. Commun. 7, 10189 (2016).

[7] P. Witham and E. Sanchez, J. Microsc. 248, 223 (2012). 
[8] A. Salvador Palau, G. Bracco, and B. Holst, Phys. Rev. A (Accepted for publication) (2016).

[9] O. Carnal, M. Sigel, T. Sleator, H. Takuma, and J. Mlynek, Phys. Rev. Lett. 67, 3231 (1991).

[10] R. B. Doak, R. E. Grisenti, S. Rehbein, G. Schmahl, J. P. Toennies, and C. Wöll, Phys. Rev. Lett. 83, 4229 (1999).

[11] M. Greve, A. Vial, J. Stamnes, and B. Holst, Opt. Express 21, 28483 (2013).

[12] L. Kipp, M. Skibowski, R. L. Johnson, R. Berndt, R. Adelung, S. Harm, and R. Seemann, Nature 414, 184 (2001).

[13] W. Fan, L. Wei, H. Zang, L. Cao, B. Zhu, X. Zhu, C. Xie, Y. Gao, Z. Zhao, and Y. Gu, Opt. Express 21, 1473 (2013).

[14] R. G. Hobbs, V. R. Manfrinato, Y. Yang, S. A. Goodman, L. Zhang, E. A. Stach, and K. K. Berggren, Nano Letters 16, 4149 (2016).

[15] A. G. Michette, Optical Systems for Soft X Rays, edited by A. G. Michette (Plenum Press, New York, 1968).

[16] R. B. Doak, in Helium Atom Scattering from Surfaces, edited by E. Hulpke (Springer Berlin / Heidelberg, 1992).

[17] B. Holst and W. Allison, Nature 390, 244 (1997).

[18] K. Fladischer and E. al., New. J. Phys. 12, 33018 (2010).

[19] P. Sutter, M. Minniti, P. Albrecht, D. Farias, R. Miranda, and E. Sutter, Appl. Phys. Lett. 99, 211907 (2011).

[20] H. C. Schewe, B. S. Zhao, G. Meijer, and W. Schöllkopf, New. J. Phys. 11, 113030 (2009).

[21] H. C. W. Beijerinck and N. F. Verster, Physica C 111, 327 (1981).

[22] G. S. Sikora, Analysis of asymptotic behavior of free jets: Prediction of Molecular Beam Instensity and Velocity Distributions, Ph.D. thesis, Princeton (1973).

[23] J. Toennies and K. Winkelmann, J. Chem. Phys. 66, 3965 (1977).

[24] T. Reisinger, G. Bracco, S. Rehbein, G. Schmahl, W. E. Ernst, and B. Holst, J. Phys. Chem A 111, 12620 (2007).

[25] L. Pedemonte and G. Bracco, J. Chem. Phys. 119, 1433 (2003).
[26] S. D. Eder, G. Bracco, T. Kaltenbacher, and B. Holst, J. Phys. Chem A 118, 4 (2014).

[27] U. Bossel, Skimming of Molecular Beams from Diverging Non-equilibrium Gas Jets, Deutsche Luft- und Raumfahrt. Forschungsbericht (Deutsche Forschungs-und Versuchsanstalt für Luft-und Raumfahrt, 1974).

[28] H. C. W. Beijerinck, R. J. F. V. Gerwen, E. R. T. Kerstel, J. F. M. Martens, E. J. W. V. Vliembergen, M. Smits, and G. H. Kaashoek, Chem. Phys. 96, 153 (1985).

[29] H. Hedgeland, A. P. Jardine, W. Allison, and J. Ellis, Rev. Sci. Instrum. 76, 123111 (2005).

[30] M. Barr, A. Fahy, A. Jardine, J. Ellis, D. Ward, D. A. Maclaren, W. Allison, and P. C. Dastoor, Nucl. Instrum. Methods B 340, 76 (2014).

[31] T. Stewart McKechnie, General Theory of Light Propagation and Imaging Through the Atmosphere, 1st ed. (Springer, 2016) p. 624.

[32] M. Young, Josa 62, 972 (1972).

[33] S. D. Eder, T. Reisinger, M. M. Greve, G. Bracco, and B. Holst, New. J. Phys. 14, 73014 (2012).

[34] Sutter Instrument, P-97 Flaming/Brown Micropipette Puller (http://www.sutter.com/MICROPIPETTE/p97.html).

[35] M. Matsuki, Company MB Scientific, Private communication, detector available on a commercial basis upon request.

[36] M. DeKieviet, D. Dubbers, M. Klein, U. Pieles, and C. Schmidt, Rev. Sci. Instrum. 71, 123301 (2000).

[37] A. R. Alderwick, A. P. Jardine, H. Hedgeland, D. A. MacLaren, W. Allison, and J. Ellis, Rev. Sci. Instrum. 79 (2008).

[38] W. Allison, Private Communication.

[39] J. Daniel, J. R. Stigliani, R. Mittra, and R. G. Semoni, J. Opt. Soc. Am. 57, 610 (1967).

[40] J. Braun, P. K. Day, J. P. Toennies, G. Witte, and E. Neher, Rev. Sci. Instrum. 68, 3001 (1997) 